\title{
Graph-based upper bounds for the probability of the union of events
}

\author{
Pierangela Veneziani* \\ Mathematics Department \\ SUNY College at Brockport \\ 350 New Campus Drive \\ Brockport, NY, US \\ pvenezia@brockport.edu
}

Submitted: Nov 27, 2007; Accepted: Jan 28, 2008; Published: Feb 11, 2008

Mathematics Subject Classifications: 60E15, 90C05

\begin{abstract}
We consider the problem of generating upper bounds for the probability of the union of events when the individual probabilities of the events as well as the probabilities of pairs of these events are known. By formulating the problem as a Linear Program, we can obtain bounds as objective function values corresponding to dual basic feasible solutions. The new upper bounds are based on underlying bipartite and threshold type graph structures.
\end{abstract}

\section{Introduction}

The Boolean probability bounding problem can be formulated as follows: let $A_{1}, \ldots, A_{n}$ be a finite set of arbitrary events in a probability space $\Omega$, and let us assume that the individual probabilities $P\left(A_{i}\right), i=1, \ldots, n$, as well as the probabilities $P\left(\bigcap_{1 \leq i_{1}<\ldots<i_{l} \leq n} A_{i_{l}}\right)$, $l=2, \ldots, m$, of up to $m$-tuples of these events are known, where $m<n$. Using this information we want to generate upper and lower bounds for the probability of a Boolean function of these events. The integer $m$ is usually referred to as the degree of these bounds.

The Boolean probability bounding scheme is a particular instance of the optimization version of the probabilistic satisfiability (PSAT) problem. The decision version of PSAT consists in determining whether, given the probabilities that $m$ logical sentences defined

${ }^{*}$ The author wishes to thank Dr. E. Boros for his continued support and her daughter Sofia for inspiration. The author would also like to acknowledge the work of the reviewer of the manuscript. 
on $n$ logical variables are true, such probability assignment is consistent. The optimization version of PSAT in concerned with determining bounds on the probability that an additional sentence is true. PSAT is known to be NP-hard (see e.g. [7]).

Both versions of PSAT were first proposed as general problem in the theory of probability by George Boole, who suggested algebraic methods to solve it.

Hailperin [5] formulated Boole's general problem as a Linear Program and showed that Boole's method is equivalent to Fourier's elimination. Kounias and Marin [8] utilized Hailperin's linear model in their work on the Boolean probability bounding scheme and generated bounds of degree two.

Dawson and Sankoff [4] proposed a sharp lower bound for the probability that at least one out of $n$ events occurs, using the first two binomial moments of the occurrences and a linear programming formulation.

Hunter [6] found an upper bound of degree two expressed in terms of underlying spanning trees, Bukszár and Prékopa [2] by means of cherry-trees, and Bukszár and Szantai [3] by means of hypercherry trees.

Let us introduce the following notations: let $\mathcal{G}_{m}=(\mathcal{V}, \mathcal{E})$ denote the hypergraph where $\mathcal{V}=\{1, \ldots, n\}$ and $\mathcal{E}=\bigcup_{k=2}^{m} \mathcal{E}_{k}$ where $\mathcal{E}_{k}=\{I \subseteq \mathcal{V}|| I \mid=k\}, k=2, \ldots, m$. Further let $\Gamma=\mathcal{V} \cup \mathcal{E}$.

For each subset $J \subseteq \mathcal{V}$ let us define the event $C_{J}=\left(\bigcap_{i \in J} A_{i}\right)\left(\bigcap_{i \in J^{c}} A_{i}^{c}\right)$ where $J^{c}=\mathcal{V} \backslash J$, and $A_{i}^{c}=\Omega \backslash A_{i}, i=1, \ldots, n$, and to each subset $J \subseteq \mathcal{V}$ let us associate a decision variable $x_{J}=\operatorname{Pr}\left(C_{J}\right)$ and a scalar $c_{J}$.

Let us further introduce the notation $p_{I}=P\left(\bigcap_{i \in I} A_{i}\right)$ where $I \in \Gamma$, and let us set $p_{\emptyset}=1$ by definition.

Let us note that the equality $\sum_{I \subseteq J \subseteq \mathcal{V}} P\left(C_{J}\right)=P\left(\bigcap_{i \in I} A_{i}\right)$ holds for all subsets $I \in$ $\Gamma \cup\{\emptyset\}$, because the $2^{n}$ (disjoint) events $C_{J}$ 's form a partition of the probability space $\Omega$. We can write this equivalently as $\sum_{I \subseteq J \subseteq \mathcal{V}} x_{J}=p_{I}$.

Finally, let $p$ denote the vector with components $p_{I} \in[0,1], I \in \Gamma \cup\{\emptyset\}$, let $x$ be the vector with components $x_{J} \in[0,1], J \subseteq \mathcal{V}$, and let $H=\left(h_{I J}\right)$ denote the incidence matrix whose entries are defined by $h_{I J}=1$ if $I \subseteq J, h_{I J}=0$ otherwise.

The matrix $H$ has $\sum_{i=0}^{m}\left(\begin{array}{c}n \\ i\end{array}\right)$ rows and $2^{n}$ columns.

In the vectors $p$ and $x$, and in the row and column indices of the matrix $H$ the order of the elements will follow the lexicographic order of the subscript sets.

The Boolean probability bounding problem can thus be restated as a linear program of the form

$$
\begin{array}{lll}
\text { Max or Min } & \sum_{J \subseteq \mathcal{V}} c_{J} x_{J} & \\
& \sum_{I \subseteq J \subseteq \mathcal{V}} h_{I J} x_{J}=p_{I} & \forall I \in \Gamma \cup\{\emptyset\} \\
& x_{J} \geq 0 & \forall J \subseteq \mathcal{V}
\end{array}
$$


or in matrix form for the maximization problem

$$
\begin{array}{ll}
\text { Max } & c^{T} x \\
\text { st } & H x=p \\
& x \geq 0
\end{array}
$$

where the vector $c$ has components $c_{J}, J \subseteq \mathcal{V}$.

In particular, if $c^{T}=[0,1, \ldots, 1]$ problems (1) and (2) provide us with bounds for the probability $P\left(A_{1} \cup \ldots \cup A_{n}\right)$ that at least one out of $n$ events occurs.

As an illustration consider for example the case $n=3, m=2, \quad c^{T}=[0,1, \ldots, 1]$, $p_{I}=0.5$ for $|I|=1, \quad p_{I}=0.25$ for $|I|=2$ :

$$
\begin{aligned}
& \operatorname{Max} \quad x_{1}+x_{2}+x_{3}+x_{12}+x_{13}+x_{23}+x_{123} \\
& x_{\emptyset}+x_{1}+x_{2}+x_{3}+x_{12}+x_{13}+x_{23}+x_{123}=1.00 \\
& \begin{array}{llll}
x_{1} & +x_{12}+x_{13}+x_{123}=0.50
\end{array} \\
& \begin{array}{llll}
x_{2} & +x_{12} & +x_{23} & +x_{123}=0.50
\end{array} \\
& \text { st } \begin{array}{llll}
x_{3} & +x_{13} & +x_{23} & +x_{123}=0.50
\end{array} \\
& x_{12}+x_{123}=0.25 \\
& x_{13}+x_{123}=0.25 \\
& x_{23}+x_{123}=0.25 \\
& x_{\emptyset}, x_{1}, x_{2}, x_{3}, x_{12}, x_{13}, x_{23}, x_{123} \geq 0 \text {. }
\end{aligned}
$$

The optimal objective function value of the maximization problem is 1 , achieved for $x_{\emptyset}=x_{12}=x_{13}=x_{23}=0$ and $x_{1}=x_{2}=x_{3}=x_{123}=0.25$.

Consider then the dual of problem (1):

$$
\begin{array}{ll}
\text { Min } & p^{T} w \\
\text { st } & H^{T} w \geq c .
\end{array}
$$

Recall that if a linear programming problem is a maximization, the objective function value corresponding to any dual feasible basis is an upper bound for its optimum value. The best bound corresponds to the optimal basis and is called sharp because no better bound can be given based on the knowledge of the vector $p$. Thus, bounds can be obtained provided that we can construct dual feasible bases.

\section{Bounds of degree 2}

Consider problem (1) for $m=2$ and cost coefficients $c^{T}=[0,1, \ldots, 1]$. The objective function then becomes $\sum_{J \subseteq \mathcal{V}} c_{J} x_{J}=\sum_{\emptyset \neq J \subseteq \mathcal{V}} x_{J}$.

In the linear program (1) we have $1+n+\left(\begin{array}{l}n \\ 2\end{array}\right)$ constraints and $2^{n}$ variables. The first constraint $\sum_{J \subseteq \mathcal{V}} x_{J}=1$ becomes superfluous because we are going to maximize the quantity $\sum_{\emptyset \neq J \subseteq \mathcal{V}} x_{J}$. If the optimum value of the maximization problem is found to be 
larger than 1 then, by taking into account the constraint $\sum_{J \subseteq \mathcal{V}} x_{J}=1$, we can trivially set the upper bound to 1 . Therefore the first row of the matrix $H$ as well as the first column corresponding to the variable $x_{\emptyset}$ can be disregarded from our formulation. In (1) we now have $n+\left(\begin{array}{l}n \\ 2\end{array}\right)$ constraints and $2^{n}-1$ variables.

As Prékopa et al. suggested in [11], it is then possible to interpret the $n+\left(\begin{array}{l}n \\ 2\end{array}\right)$ components of any dual feasible solution $w=\left(w_{\gamma}\right)_{\gamma \in \Gamma}$ of problem (2) as node and edge weights in $\mathcal{G}_{2}$, that is a weight $w_{i}$ is assigned to node $i \in \mathcal{V}$ and a weight $w_{i, j}$ is assigned to edge $\{i, j\} \in \mathcal{E}_{2}$.

In what follows we will let $\mathcal{E}(S)$ denote the edge set of a subset $S \subseteq \mathcal{V}$ and $w(S)=$ $\sum_{\gamma \in S} w_{\gamma}+\sum_{\gamma \in \mathcal{E}(S)} w_{\gamma}$ represent the weight of subset $S$ for a given dual feasible solution $w=\left(w_{\gamma}\right)_{\gamma \in \Gamma}$.

For the instance under study $\left(c^{T}=[0,1, \ldots, 1]\right.$ and $\left.m=2\right)$ problem $(2)$ can then be written as

$$
\begin{array}{ll}
\text { Min } & \sum_{\gamma \in \Gamma} p_{\gamma} w_{\gamma} \\
\text { st } & w(S) \geq 1 \quad \forall S \subseteq \mathcal{V} .
\end{array}
$$

The lemma that follows provides a sufficient and necessary condition for a given vector to be a basic feasible solution of problem (3) by use of the graph structure introduced at the beginning of section 2 .

Lemma 1 Given a collection $\Im=\left\{I_{\gamma}\right\}_{\gamma \in \Gamma}$ of column subscripts of the matrix $H$, a vector $w=\left(w_{\gamma}\right)_{\gamma \in \Gamma}$ is a dual basic feasible solution of problem (1) generated by the basis $\Im$ if the following conditions are satisfied.

(i) The vector $w=\left(w_{\gamma}\right)_{\gamma \in \Gamma}$ is the unique solution of the system of equations $w\left(I_{\gamma}\right)=1$ for all subsets $I_{\gamma} \in \Im, \gamma \in \Gamma$.

(ii) For all subsets $S \subseteq \mathcal{V}$ such that $S \notin \Im$ the inequality $w(S) \geq 1$ holds.

Proof. Let $h_{J}, J \subseteq \mathcal{V}$, designate a column vector of the matrix $H$. Let $B$ denote a nonsingular square submatrix of $H$ of order $m$ and let $\Im=\left\{I_{\gamma}\right\}_{\gamma \in \Gamma}$ denote the collection of subscripts whose columns form $B$. Recall that a matrix $B$ is said to be a dual feasible basis of problem (1) if $c_{B}^{T} B^{-1} h_{I_{\gamma}}=c_{I_{\gamma}}$ for all subsets $I_{\gamma} \in \Im, \gamma \in \Gamma$, and $c_{B}^{T} B^{-1} h_{J} \geq c_{J}$ for all subsets $J \notin \Im$. The corresponding dual basic feasible solution is the vector $w^{T}=c_{B}^{T} B^{-1}$.

In our case, condition $(i)$ guarantees that the matrix $B$ is nonsingular and that the equalities $c_{B}^{T} B^{-1} h_{I_{\gamma}}=c_{I_{\gamma}}$ hold for all basic sets $I_{\gamma} \in \Im, \gamma \in \Gamma$, and condition ( $i i$ ) ensures that the inequalities $c_{B}^{T} B^{-1} h_{J} \geq c_{J}$ are satisfied for all nonbasic sets $J \notin \Im$.

Remark 2 Let $\mathcal{G}^{*}=\left(\Gamma \cup \Im, \mathcal{E}^{*}\right)$ denote the bipartite graph where $\mathcal{E}^{*}=\{I \in \Gamma, J \in \Im \mid$ $I \subseteq J\}$. A necessary condition for a collection $\Im=\left\{I_{\gamma}\right\}_{\gamma \in \Gamma}$ of column subscripts of $H$ to form a basis is that there exists a perfect matching in the bipartite graph $\mathcal{G}^{*}$, otherwise if no perfect matching exists the matrix B would be singular (see e.g. [10]). Therefore in constructing a basis $\Im=\left\{I_{\gamma}\right\}_{\gamma \in \Gamma}$ we want to make sure to cover all the nodes and edges of $\mathcal{G}_{2}$. 
The new families of dual feasible bases presented in the propositions that follow are obtained by partitioning the graph $\mathcal{G}_{2}$ in two components and then assigning nonnegative weights to each component and nonpositive weights to the edges connecting them.

Proposition 3 Assume $n \geq 5$. Let $\mathcal{V}=\mathcal{A} \cup \mathcal{B}$ be a partition of the vertex set $\mathcal{V}$ and $k=|\mathcal{A}|$ where $2 \leq k \leq\left\lfloor\frac{n}{2}\right\rfloor$. Let $l$ denote a positive integer such that $k \leq l \leq n-k-1$, $\left(\begin{array}{c}n-k \\ 2\end{array}\right) \leq\left(\begin{array}{c}n-k \\ l\end{array}\right)$, and $\left(\begin{array}{c}k \\ 2\end{array}\right) \leq\left(\begin{array}{c}n-k \\ l+1\end{array}\right)$ Then the vector $w=\left(w_{\gamma}\right)_{\gamma \in \Gamma}$ with components

$$
w_{\gamma}=\left\{\begin{array}{lll}
1 & \text { if } \gamma \in \mathcal{V} \\
-1 & \text { if } \gamma=\{i, j\}, \quad i \in \mathcal{A}, \quad j \in \mathcal{B} \\
\frac{l-1}{k} & \text { if } \gamma=\{i, j\}, \quad i \in \mathcal{A}, \quad j \in \mathcal{A} \\
\frac{k-1}{l} \quad \text { if } \gamma=\{i, j\}, \quad i \in \mathcal{B}, \quad j \in \mathcal{B}
\end{array}\right.
$$

is a dual basic feasible solution of problem (1).

Proof. Let $\mathcal{A}_{s} \subseteq \mathcal{A}\left(\mathcal{B}_{r} \subseteq \mathcal{B}\right)$ denote a subset of cardinality $s, 1 \leq s \leq k \quad(r$, $1 \leq r \leq n-k)$.

Define $\Im=\left\{I_{\gamma}\right\}_{\gamma \in \Gamma}$ to be the collection of column labels of the matrix $H$ where

$$
\begin{aligned}
I_{i} & =\{i\} \quad \text { for } i \in \mathcal{V} \\
I_{i, j} & = \begin{cases}\{i, j\} & \text { if } i \in \mathcal{A}, j \in \mathcal{B} \\
\mathcal{A} \cup \mathcal{B}_{l+1} & \text { if } i, j \in \mathcal{A} \\
\mathcal{A} \cup \mathcal{B}_{l} & \text { if } i, j \in \mathcal{B}, \text { where } i, j \in \mathcal{B}_{l} .\end{cases}
\end{aligned}
$$

The vector $w$ is a dual basic feasible solution of problem (1) generated by the basis $\checkmark$ because conditions $(i)$ and (ii) of lemma 1 are met, as shown below.

(i) For all $i \in \mathcal{V} \quad w\left(I_{i}\right)=1$ if and only if $w_{i}=1$.

For all $\{i, j\} \in \mathcal{E}_{2}$ such that $i \in \mathcal{A}, j \in \mathcal{B} w\left(I_{i, j}\right)=w(\{i, j\})=w_{i}+w_{j}+w_{i, j}=$ $2+w_{i, j}=1$ if and only if $w_{i, j}=-1$.

The symmetry of the constraints ensures that $w_{h, k}=x$ for all $h, k \in \mathcal{A}$ and $w_{h, k}=y$ for all $h, k \in \mathcal{B}$.

Then for all $\{i, j\} \in \mathcal{E}(\mathcal{A})$

$$
\begin{aligned}
w\left(I_{i, j}\right)=w\left(\mathcal{A} \cup \mathcal{B}_{l+1}\right) & =\sum_{f \in \mathcal{A} \cup \mathcal{B}_{l+1}} w_{f}+\sum_{f, g \in \mathcal{A}} w_{f, g}+\sum_{f, g \in \mathcal{B}_{l+1}} w_{f, g}+\sum_{f \in \mathcal{A}, g \in \mathcal{B}_{l+1}} w_{f, g} \\
& =(k+l+1)(1)+\left(\begin{array}{c}
k \\
2
\end{array}\right) x+\left(\begin{array}{c}
l+1 \\
2
\end{array}\right) y+k(l+1)(-1)=1
\end{aligned}
$$

if and only if

$$
\frac{k(k-1)}{2} x+\frac{(l+1) l}{2} y=k l-l
$$

For all $\{i, j\} \in \mathcal{E}(\mathcal{B})$

$$
\begin{aligned}
w\left(I_{i, j}\right)=w\left(\mathcal{A} \cup \mathcal{B}_{l}\right) & =\sum_{f \in \mathcal{A} \cup \mathcal{B}_{l}} w_{f}+\sum_{f, g \in \mathcal{A}} w_{f, g}+\sum_{f, g \in \mathcal{B}_{l}} w_{f, g}+\sum_{f \in \mathcal{A}, g \in \mathcal{B}_{l}} w_{f, g} \\
& =(k+l)(1)+\left(\begin{array}{l}
k \\
2
\end{array}\right) x+\left(\begin{array}{l}
l \\
2
\end{array}\right) y+k l(-1)=1
\end{aligned}
$$


if and only if

$$
\frac{k(k-1)}{2} x+\frac{l(l-1)}{2} y=k l-k-l+1 .
$$

The unique solution of the system of equations $I-I I$ is given by $x=\frac{l-1}{k}$ and $y=\frac{k-1}{l}$.

(ii) The only nontrivial case that needs to be considered to prove that the vector $w$ is feasible for problem (3) is $S=\mathcal{A}_{s} \cup \mathcal{B}_{r}, 1 \leq s \leq k, 1 \leq r \leq n-k$.

Then

$$
\begin{aligned}
w(S)=w\left(\mathcal{A}_{s} \cup \mathcal{B}_{r}\right) & =\sum_{f \in \mathcal{A}_{s} \cup \mathcal{B}_{r}} w_{f}+\sum_{f, g \in \mathcal{A}_{s}} w_{f, g}+\sum_{f, g \in \mathcal{B}_{r}} w_{f, g}+\sum_{f \in \mathcal{A}_{s}, g \in \mathcal{B}_{r}} w_{f, g} \\
& =s+r+\left(\begin{array}{l}
s \\
2
\end{array}\right) x+\left(\begin{array}{l}
r \\
2
\end{array}\right) y+r s(-1) \\
& =\frac{y r^{2}+r(2-2 s-y)+x s^{2}-x s+2 s}{2} .
\end{aligned}
$$

Consider the following cases.

Case 1: $s=1$. Then $w\left(\mathcal{A}_{1} \cup \mathcal{B}_{r}\right)=1+\frac{y r(r-1)}{2} \geq 1$ because $r \geq 1$ and $y \geq 0$.

Case 2: $s=k$. Then $w\left(\mathcal{A} \cup \mathcal{B}_{r}\right)=\frac{r^{2}-r(1+2 l)+l(l+1)+2}{2}$. Therefore the inequality $w(\mathcal{A} \cup$ $\left.\mathcal{B}_{r}\right) \geq 1$ holds if and only if $r^{2}-r(1+2 l)+l(l+1) \geq 0$ equivalently $r \leq l$ or $r \geq l+1$.

Case 3: $2 \leq s \leq k-1$. Consider the polynomial

$$
\mathcal{P}(r \mid s, l)=w(S)-1=\frac{y r^{2}+r(2-2 s-y)+x s^{2}-x s+2 s}{2}-1
$$

where $r \in[1, n-k]$, and let $\Delta(s, l)$ denote the discriminant of the equation $\mathcal{P}(r \mid s, l)=0$.

We will show that $\Delta(s, l)<0$ for every $s \in[2, k-1], l \in[k, n-k-1]$, thus $\mathcal{P}(r \mid s, l)>0$ for any $r$, equivalently $w\left(\mathcal{A}_{s} \cup \mathcal{B}_{r}\right) \geq 1$ for any subset $\mathcal{A}_{s} \cup \mathcal{B}_{r} \subseteq \mathcal{V}$.

The expression of the discriminant is given by

$$
\begin{aligned}
\Delta(s, l) & =(2-2 s-y)^{2}-4 y\left(x s^{2}-x s+2 s-2\right) \\
& =\frac{4(1-s)(k-s) l^{2}+4(k-1)(1-s)(k-s) l+k(k-1)^{2}}{k l^{2}} .
\end{aligned}
$$

The inequality $\Delta(s, l)<0$ can be written as $\Delta^{*}(s, l)=-k l^{2} \Delta(s, l)>0$.

Because the partial derivative of the function $\Delta^{*}(s, l)$ with respect to the variable $l$

$$
\frac{\partial \Delta^{*}(s, l)}{\partial l}=8(s-1)(k-s) l+4(k-1)(s-1)(k-s)=4(s-1)(k-s)(2 l+k-1)
$$

is positive when $l \geq-\left(\frac{k-1}{2}\right)$, the function $\Delta^{*}(s, l)$ is increasing in $l$ on the interval $[k, n-k-1]$. If we show that the inequality $\Delta^{*}(s, l=k) \geq 0$ is satisfied for any $s \in[2, k-1]$, the argument will be complete.

Because $\Delta^{*}(s, l=k)=k\left[4(1-2 k) s^{2}+4\left(2 k^{2}+k-1\right) s-(3 k-1)^{2}\right]$, the sign of the first derivative $\frac{d\left(\Delta^{*}(s, l=k)\right)}{d s}=k\left[8(1-2 k) s+4\left(2 k^{2}+k-1\right)\right]$ is nonnegative for $s \leq 4(k+1)$, that is the function $\Delta^{*}(s, l=k)$ is increasing in $s$ on the interval $[2, k-1]$. 
Therefore to conclude that the function $\Delta^{*}(s, l=k)$ is nonnegative on the interval $s \in[2, k-1]$ it then suffices to notice that $\Delta^{*}(s=2, l=k)=7 k^{2}-18 k+7 \geq 13>0$, because in the case under study $k \geq 3$.

The bound generated by evaluating the objective function of problem (3) at the dual basic feasible solution described by the above proposition is given by

$$
P\left(\bigcup_{i=1}^{n} A_{i}\right) \leq \sum_{j \in \mathcal{V}} p_{j}-\sum_{i \in \mathcal{A}, j \in \mathcal{B}} p_{i, j}+\frac{l-1}{k} \sum_{i, j \in \mathcal{A}} p_{i, j}+\frac{k-1}{l} \sum_{i, j \in \mathcal{B}} p_{i, j} .
$$

Remark 4 The assumptions $k \leq l \leq n-k-1,\left(\begin{array}{c}n-k \\ 2\end{array}\right) \leq\left(\begin{array}{c}n-k \\ l\end{array}\right)$, and $\left(\begin{array}{c}k \\ 2\end{array}\right) \leq\left(\begin{array}{c}n-k \\ l+1\end{array}\right)$ guarantee that there is a sufficient number of sets needed to form a basis as described in the above proof. Moreover $l=k$ is the smallest value for which the proposition holds true.

Proposition 5 Assume $n \geq 5$. Let $\mathcal{V}=\mathcal{A} \cup \mathcal{B}$ be a partition of the vertex set $\mathcal{V}$ and $l=|\mathcal{A}|$ where $1 \leq l \leq n-1$. Then the vector $w=\left(w_{\gamma}\right)_{\gamma \in \Gamma}$ with components

$$
w_{\gamma}= \begin{cases}1 & \text { if } \gamma \in \mathcal{V} \\ -1 & \text { if } \gamma=\{i, j\}, \quad i \in \mathcal{A}, j \in \mathcal{B} \\ 0 & \text { if } \gamma=\{i, j\}, \quad i, j \in \mathcal{A} \\ l-1 & \text { if } \gamma=\{i, j\}, i, j \in \mathcal{B}\end{cases}
$$

is a dual basic feasible solution of problem (1).

Proof. Define $\Im=\left\{I_{\gamma}\right\}_{\gamma \in \Gamma}$ to be the collection of column labels of the matrix $H$ where

$$
\begin{aligned}
I_{i} & =\{i\} \text { for } i \in \mathcal{V} \\
I_{i, j} & = \begin{cases}\{i\} \cup\{j\} & \text { if } i \in \mathcal{A}, j \in \mathcal{B} \\
\{i\} \cup\{j\} \cup\{k\} & \text { if } i, j \in \mathcal{A}, \text { where } k \in \mathcal{B} \\
\{i\} \cup\{j\} \cup \mathcal{A} & \text { if } i, j \in \mathcal{B}\end{cases}
\end{aligned}
$$

The vector $w$ is a dual basic feasible solution of problem (1) generated by the basis $\Im$ because conditions $(i)$ and (ii) of lemma 1 are met, as shown below.

(i) For all $i \in \mathcal{V} \quad w\left(I_{i}\right)=1$ if and only if $w_{i}=1$.

For all $\{i, j\} \in \mathcal{E}_{2}$ with $i \in \mathcal{A}, j \in \mathcal{B} w\left(I_{i, j}\right)=w(\{i\} \cup\{j\})=w_{i}+w_{j}+w_{i, j}=2+w_{i, j}=1$ if and only if $w_{i, j}=-1$.

For all $\{i, j\} \in \mathcal{E}(\mathcal{A})$

$$
w\left(I_{i, j}\right)=w(\{i\} \cup\{i\} \cup\{k\})=w_{i}+w_{j}+w_{k}+w_{i, j}+w_{i, k}+w_{j, k}=3-2+w_{i, j}=1
$$

if and only if $w_{i, j}=0$.

Finally for all $i, j \in \mathcal{E}(\mathcal{B})$

$$
\begin{aligned}
w\left(I_{i, j}\right)=w(\{i\} \cup\{j\} \cup \mathcal{A}) & =\sum_{h \in\{i\} \cup\{j\} \cup \mathcal{A}} w_{h}+\sum_{h \in \mathcal{A}} w_{h, i}+\sum_{h \in \mathcal{A}} w_{h, j}+\sum_{h, k \in \mathcal{A}} w_{h, k}+w_{i, j} \\
& =l+2+2 l(-1)+\left(\begin{array}{l}
l \\
2
\end{array}\right)(0)+w_{i, j} \\
& =2-l+w_{i, j}=1
\end{aligned}
$$


if and only if $w_{i, j}=l-1$.

(ii) The only nontrivial case that need to be considered to prove that the vector $w$ is feasible for problem (3) is $S=\mathcal{A}_{s} \cup \mathcal{B}_{r}$, where $\mathcal{A}_{s} \subseteq \mathcal{A},\left|\mathcal{A}_{s}\right|=s, 1 \leq s \leq l$, and $\mathcal{B}_{r} \subseteq \mathcal{B}$, $\left|\mathcal{B}_{r}\right|=r, \quad 1 \leq r \leq n-l$. Then

$$
\begin{aligned}
w\left(\mathcal{A}_{s} \cup \mathcal{B}_{r}\right) & =\sum_{f \in \mathcal{A}_{s} \cup \mathcal{B}_{r}} w_{f}+\sum_{f, g \in \mathcal{A}_{s}} w_{f, g}+\sum_{f, g \in \mathcal{B}_{r}} w_{f, g}+\sum_{f \in \mathcal{A}_{s}, g \in \mathcal{B}_{r}} w_{f, g} \\
& =s+r+(l-1)\left(\begin{array}{l}
r \\
2
\end{array}\right)-s r \\
& =\frac{(l-1) r^{2}-r(2 s-2+l-1)+2 s}{2} .
\end{aligned}
$$

Therefore the inequality $w\left(\mathcal{A}_{s} \cup \mathcal{B}_{r}\right) \geq 1$ holds if and only if $(l-1) r^{2}-r(2 s-2+l-$ 1) $+2 s-2 \geq 0$ equivalently $r \leq \frac{s-2}{l-1}$ or $r \geq 1$, since $\frac{s-2}{l-1}<1$ because $s<l+1$.

The bound generated by evaluating the objective function of problem (3) at the basic feasible solution described by the above proposition is given by

$$
P\left(\bigcup_{i=1}^{n} A_{i}\right) \leq \sum_{i \in \mathcal{V}} p_{i}+(l-1) \sum_{i, j \in \mathcal{B}} p_{i, j}-\sum_{i \in \mathcal{A}, j \in \mathcal{B}} p_{i, j} .
$$

The following corollary shows that a known bound [11] can be obtained as a special case of the bound presented in proposition 7 .

Corollary 6 Assume $n \geq 5$. For fixed $i_{1}, i_{2} \in \mathcal{V}$, let $\mathcal{A}=\mathcal{V} \backslash\left\{\left\{i_{1}\right\} \cup\left\{i_{2}\right\}\right\}$. Then the vector $w=\left(w_{\gamma}\right)_{\gamma \in \Gamma}$ with components

$$
w_{\gamma}= \begin{cases}1 & \text { if } \gamma \in \mathcal{V} \\ -1 & \text { if } \gamma=\left\{i_{k}, j\right\}, k=1,2, j \in \mathcal{A} \\ 0 & \text { if } \gamma=\{i, j\}, \quad i, j \in \mathcal{A} \\ n-3 & \text { if } \gamma=\left\{i_{1}, i_{2}\right\}\end{cases}
$$

is a dual basic feasible solution of problem (1).

Proof. Set $\mathcal{B}=\left\{i_{1}, i_{2}\right\}$ in proposition 6 .

We conclude the section presenting a new bound that is obtained by means of an underlying threshold-type graph structure.

Proposition 7 Assume $n \geq 5$. Let $\mathcal{V}=\mathcal{A} \cup \mathcal{B}$ be a partition of the vertex set $\mathcal{V}$. Let $s=|\mathcal{A}|, 1 \leq s \leq n-1$, and $\mathcal{A}=\left\{a_{1}, a_{2}, \ldots, a_{s}\right\}$.

Let $\mathcal{N}\left(a_{k}\right), k=1, \ldots, s$, denote the set of vertices $i \in \mathcal{B}$ that are connected to vertex $a_{k}$. Assume that $\mathcal{N}\left(a_{1}\right)=\mathcal{B}, \mathcal{N}\left(a_{h}\right) \subseteq \mathcal{N}\left(a_{k}\right)$ if $h>k$, and $\mathcal{N}\left(a_{h}\right) \cap \mathcal{N}\left(a_{k}\right) \neq \emptyset$ for all $h, k=1, \ldots, s$. Then the vector $w=\left(w_{\gamma}\right)_{\gamma \in \Gamma}$ with component

$$
w_{\gamma}= \begin{cases}1 & \text { if } \gamma \in \mathcal{V} \\ -1 & \text { if } \gamma=\left\{a_{k}, j\right\}, j \in \mathcal{N}\left(a_{k}\right), 1 \leq k \leq s \\ \left|\mathcal{N}\left(a_{h}\right) \cap \mathcal{N}\left(a_{k}\right)\right|-1 & \text { if } \gamma=\left\{a_{h}, a_{k}\right\}, \quad 1 \leq h, k \leq s \\ 0 & \text { otherwise }\end{cases}
$$

is a dual basic feasible solution of problem (1). 
Proof. Define $\Im=\left\{I_{\gamma}\right\}_{\gamma \in \Gamma}$ to be the collection of column labels of the matrix $H$ where

$$
\begin{aligned}
& I_{i}=\{i\} \quad \text { for } i \in \mathcal{V} \\
& I_{i, j}= \begin{cases}\left\{a_{k}, j\right\} & \text { if } i=a_{k}, j \in \mathcal{N}\left(a_{k}\right), 1 \leq k \leq s \\
\left\{\mathcal{N}\left(a_{h}\right) \cap \mathcal{N}\left(a_{k}\right)\right\} \cup\left\{a_{h}\right\} \cup\left\{a_{k}\right\} & \text { if } i=a_{h}, j=a_{k}, 1 \leq h, k \leq s \\
\left\{a_{1}\right\} \cup\{i\} \cup\{j\} & \text { if } i, j \in \mathcal{B} \\
\left\{a_{h}\right\} \cup\left\{a_{k}\right\} \cup\{j\} \cup \mathcal{N}\left(a_{k}\right) & \text { if } i=a_{k}, j \in \mathcal{B} \backslash \mathcal{N}\left(a_{k}\right), 1 \leq k \leq s \\
& \text { where } h=\max \left\{t \mid j \in \mathcal{N}\left(a_{t}\right)\right\} .\end{cases}
\end{aligned}
$$

The vector $w$ is a dual basic feasible solution of problem (1) generated by the basis $\Im$ because conditions $(i)$ and (ii) of lemma 1 are met, as shown below.

(i) For all $i \in \mathcal{V} w\left(I_{i}\right)=1$ if and only if $w_{i}=1$.

For all $\left\{a_{k}, j\right\} \in \mathcal{E}_{2}$ with $j \in \mathcal{N}\left(a_{k}\right), 1 \leq k \leq s$,

$$
w\left(I_{a_{k}, j}\right)=w\left(\left\{a_{k}, j\right\}\right)=w_{a_{k}}+w_{j}+w_{a_{k}, j}=2+w_{a_{k}, j}=1
$$

if and only if $w_{a_{k}, j}=-1$.

For all $\{i, j\} \in \mathcal{E}(\mathcal{B})$

$$
w\left(I_{i, j}\right)=w\left(\left\{a_{1}\right\} \cup\{i\} \cup\{j\}\right)=w_{a_{1}}+w_{i}+w_{j}+w_{a_{1}, i}+w_{a_{1}, j}+w_{i, j}=3-2+w_{i, j}=1
$$

if and only if $w_{i, j}=0$.

For $\{i, j\}=\left\{a_{h}, a_{k}\right\}, \quad 1 \leq h, k \leq s$,

$$
\begin{aligned}
w\left(I_{a_{h}, a_{k}}\right)= & w\left(\left\{\mathcal{N}\left(a_{h}\right) \cap \mathcal{N}\left(a_{k}\right)\right\} \cup\left\{a_{h}\right\} \cup\left\{a_{k}\right\}\right) \\
= & \sum_{f \in\left\{\mathcal{N}\left(a_{h}\right) \cap \mathcal{N}\left(a_{k}\right)\right\} \cup\left\{a_{h}\right\} \cup\left\{a_{k}\right\}} w_{f}+\sum_{f \in \mathcal{N}\left(a_{h}\right) \cap \mathcal{N}\left(a_{k}\right)} w_{a_{h}, f}+\sum_{f \in \mathcal{N}\left(a_{h}\right) \cap \mathcal{N}\left(a_{k}\right)} w_{a_{k}, f} \\
& +\sum_{f, g \in \mathcal{N}\left(a_{h}\right) \cap \mathcal{N}\left(a_{k}\right)} w_{f, g}+w_{a_{h}, a_{k}} \\
= & \left|\mathcal{N}\left(a_{h}\right) \cap \mathcal{N}\left(a_{k}\right)\right|+2+\left|\mathcal{N}\left(a_{h}\right) \cap \mathcal{N}\left(a_{k}\right)\right|(-1)+w_{a_{h}, a_{k}} \\
& +\left|\mathcal{N}\left(a_{h}\right) \cap \mathcal{N}\left(a_{k}\right)\right|(-1)+\left(\begin{array}{c}
\left|\mathcal{N}\left(a_{h}\right) \cap \mathcal{N}\left(a_{k}\right)\right| \\
2
\end{array}\right)(0) \\
= & 2-\left|\mathcal{N}\left(a_{h}\right) \cap \mathcal{N}\left(a_{k}\right)\right|+w_{a_{h}, a_{k}}=1
\end{aligned}
$$

if and only if $w_{a_{h}, a_{k}}=\left|\mathcal{N}\left(a_{h}\right) \cap \mathcal{N}\left(a_{k}\right)\right|-1$.

Finally for $\left\{a_{k}, j\right\} \in \mathcal{E}_{2}$ with $j \in \mathcal{B} \backslash \mathcal{N}\left(a_{k}\right), 1 \leq k \leq s$,

$$
\begin{aligned}
w\left(I_{a_{k}, j}\right)= & w\left(\left\{a_{h}\right\} \cup\left\{a_{k}\right\} \cup\{j\} \cup \mathcal{N}\left(a_{k}\right)\right) \\
= & w_{a_{h}}+w_{a_{k}}+w_{j}+\sum_{f \in \mathcal{N}\left(a_{k}\right)} w_{f}+w_{a_{h}, a_{k}}+w_{a_{h}, j}+w_{a_{k}, j} \\
& +\sum_{f \in \mathcal{N}\left(a_{k}\right)} w_{a_{h}, f}+\sum_{f \in \mathcal{N}\left(a_{k}\right)} w_{a_{k}, f}+\sum_{f \in \mathcal{N}\left(a_{k}\right)} w_{j, f} \\
= & 3+\left|\mathcal{N}\left(a_{k}\right)\right|+\left|\mathcal{N}\left(a_{h}\right) \cap \mathcal{N}\left(a_{k}\right)\right|-1-1+w_{a_{k}, j} \\
& +\left|\mathcal{N}\left(a_{k}\right)\right|(-1)+\left|\mathcal{N}\left(a_{k}\right)\right|(-1)+\left|\mathcal{N}\left(a_{k}\right)\right|(0) \\
= & 1+w_{a_{k}, j}=1
\end{aligned}
$$


if and only if $w_{a_{k}, j}=0$, since $\left|\mathcal{N}\left(a_{h}\right) \cap \mathcal{N}\left(a_{k}\right)\right|=\left|\mathcal{N}\left(a_{k}\right)\right|$ because $k>h$.

(ii) The only nontrivial case that needs to be considered to prove that the vector $w$ is feasible for problem (2) is $S=C \cup D$, where $C \subseteq \mathcal{A}$ and $D \subseteq \mathcal{B}$. We will show that the inequality $w(C \cup D) \geq 1$ holds by induction on $|C|$.

If $|C|=1$ let $C=\left\{a_{i_{1}}\right\}$, where $1 \leq i_{1} \leq s$. Then

$$
\begin{aligned}
w(S)=w\left(\left\{a_{i_{1}}\right\} \cup D\right) & =\sum_{j \in\left\{a_{i_{1}}\right\} \cup D} w_{j}+\sum_{j \in D} w_{a_{i_{1}}, j}+\sum_{i, j \in D} w_{i, j} \\
& =1+|D|+\left|\mathcal{N}\left(a_{i_{1}}\right) \cap D\right|(-1)+0 \geq 1
\end{aligned}
$$

because $\left|\mathcal{N}\left(a_{i_{1}}\right) \cap D\right| \leq|D|$.

Let us assume that inequality $w(C \cup D) \geq 1$ holds for $|C|=k$.

If $|C|=k+1$ let $C=\left\{a_{i_{1}}, a_{i_{2}}, \ldots, a_{i_{k}}, a_{i_{k+1}}\right\}$, where $1 \leq i_{1}<i_{2}<\ldots<i_{k}<i_{k+1} \leq s$, then

$$
\begin{aligned}
w(S)= & w\left(\left\{a_{i_{1}}, a_{i_{2}}, \ldots, a_{i_{k+1}}\right\} \cup D\right) \\
= & w\left(\left\{a_{i_{1}}, a_{i_{2}}, \ldots, a_{i_{k}}\right\} \cup D\right)+w_{a_{i_{k+1}}}+\sum_{j \in D} w_{a_{i_{k+1}}, j}+\sum_{l=1}^{k} w_{a_{i_{l}}, a_{i_{k+1}}} \\
= & w\left(\left\{a_{i_{1}}, a_{i_{2}}, \ldots, a_{i_{k}}\right\} \cup D\right)+1+\left|\mathcal{N}\left(a_{i_{k+1}}\right) \cap D\right|(-1) \\
& +\sum_{l=1}^{k}\left[\left|\mathcal{N}\left(a_{i_{k+1}}\right) \cap \mathcal{N}\left(a_{i_{l}}\right)\right|-1\right] \\
= & w\left(\left\{a_{i_{1}}, a_{i_{2}}, \ldots, a_{i_{k}}\right\} \cup D\right)+1-\left|\mathcal{N}\left(a_{i_{k+1}}\right) \cap D\right|+\left|\mathcal{N}\left(a_{i_{k+1}}\right) \cap \mathcal{N}\left(a_{i_{1}}\right)\right|-1 \\
& +\sum_{l=2}^{k}\left[\left|\mathcal{N}\left(a_{i_{k+1}}\right) \cap \mathcal{N}\left(a_{i_{l}}\right)\right|-1\right] \\
= & w\left(\left\{a_{i_{1}}, a_{i_{2}}, \ldots, a_{i_{k}}\right\} \cup D\right)+\left|\mathcal{N}\left(a_{i_{k+1}}\right) \cap \mathcal{N}\left(a_{i_{1}}\right)\right| \\
& -\left|\mathcal{N}\left(a_{i_{k+1}}\right) \cap D\right|+\sum_{l=2}^{k}\left[\left|\mathcal{N}\left(a_{i_{k+1}}\right) \cap \mathcal{N}\left(a_{i_{l}}\right)\right|-1\right] \geq 1
\end{aligned}
$$

because $w\left(\left\{a_{i_{1}}, a_{i_{2}}, \ldots, a_{i_{k}}\right\} \cup D\right) \geq 1$ by the induction hypothesis and $\left|\mathcal{N}\left(a_{i_{k+1}}\right) \cap \mathcal{N}\left(a_{i_{1}}\right)\right|-$ $\left|\mathcal{N}\left(a_{i_{k+1}}\right) \cap D\right| \geq 0$ since $\left|\mathcal{N}\left(a_{i_{k+1}}\right) \cap \mathcal{N}\left(a_{i_{1}}\right)\right|=\left|\mathcal{N}\left(a_{i_{k+1}}\right)\right| \geq\left|\mathcal{N}\left(a_{i_{k+1}}\right) \cap D\right|$.

The bound generated by evaluating the objective function of problem (3) at the dual basic feasible solution described by the above proposition is given by

$$
P\left(\bigcup_{i=1}^{n} A_{i}\right) \leq \sum_{i \in \mathcal{V}} p_{i}+\sum_{\substack{h, k=1 \\ h \neq k}}^{s}\left[\left|\mathcal{N}\left(a_{h}\right) \cap \mathcal{N}\left(a_{k}\right)\right|-1\right] p_{a_{h}, a_{k}}-\sum_{k=1}^{s} \sum_{j \in \mathcal{N}\left(a_{k}\right)} p_{a_{k}, j} .
$$

Finally we will compare one of the new bounds with Kwerel's bound [9] and Hunter's bound [6] for the system II in [1], for which $n=6, P\left(\bigcup_{i=1}^{n} A_{i}\right)=0.6740$, 
$p_{1}=0.268, p_{2}=0.312, p_{3}=0.302, p_{4}=0.172, p_{5}=0.384, p_{6}=0.278$,

$p_{12}=0, p_{13}=0.168, p_{14}=0.033, p_{15}=0.19, p_{16}=0.155$,

$p_{23}=0.078, p_{24}=0.045, p_{25}=0.156, p_{26}=0.067$,

$p_{34}=0.056, p_{35}=0.201, p_{36}=0.111$,

$p_{45}=0.049, p_{46}=0.089, p_{56}=0.189$.

Numerical results for the given system are given in the table below.

Upper Bounds of Degree 2

$\begin{array}{cc}\text { Kwerel's bound } & 1 \\ \text { Hunter's bound } & 0.891 \\ \text { Our bound* } & 0.813 \\ \text { Exact value } & 0.674\end{array}$

*Our bound was generated by setting $\mathcal{A}=\{1,2,4\}, \mathcal{B}=\{3,5,6\}$, and $l=1$ in proposition 6. It is possible to show that our bound is the best possible bound that can be generated via the linear programming formulation of the Boolean Probability Bounding Problem for the numerical example under study.

\section{References}

[1] F. Alajaji, H. Kuai and G. Takahara, A Lower Bound for the Probability of a Finite Union of Events Discrete Applied Mathematics, 215 (2000), 147 - 158.

[2] J. Bukszár and A. Prékopa, Probability bounds with cherry trees, Math. Oper. Res., 26 (2001), 174 - 192.

[3] J. Bukszár and T. Szántai, Probability bounds given by hypercherry trees, Alkalmaz. Mat. Lapok, 19 (1999), 69 - 85.

[4] D.A. Dawson and S. Sankoff, An Inequality for Probability, . Proc. Am. Math. Soc., 18 (1967), 504-507.

[5] T. Hailperin, Best possible inequalities for the probability of a logical function of events, The American Mathematical Monthly, 72 (1965), 343 - 359.

[6] D. Hunter, An upper bound for the probability of the union, J. Appl. Prob., 30 (1975), $597-603$.

[7] B. Jaumard, P. Hansen and M. Poggi de Aragão, Column generation methods for probabilistic logic, ORSA J. Comput., 3 (1991), 135 - 148.

[8] S. Kounias and J. Marin, Best linear bonferroni bounds, SIAM J. on Applied Mathematics, 30 (1976), 301 - 326.

[9] S.M. Kwerel, Most stringent bounds on aggregated probabilities of partially specified dependent probability systems, J. Am. Statist. Assoc. 70 (1975) 472-479.

[10] L. Lovász and M.D. Plummer, Matching theory, Ann. Discrete Math., 29 (1986).

[11] A. Prékopa, B. Vizvári and G. Regös, Lower and upper bounds on probabilities of Boolean functions of events, Rutcor Research Report, 36-95 (1995) 